\title{
Maximal inspiratory and expiratory pressure measurement in tracheotomised patients
}

\author{
M. Vitacca*, M. Paneroni*, L. Bianchi*, E. Clini ${ }^{\star}$, A. Vianello ${ }^{+}$, P. Ceriana ${ }^{\#}$, \\ L. Barbano*, B. Balbi* and S. Nava*
}

ABSTRACT: The present study compared four different sites and conditions for the measurement of maximal inspiratory pressure (MIP) and maximal expiratory pressure (MEP) in 38 spontaneous breathing tracheotomised patients. Of the patients, 28 had chronic obstructive pulmonary disease (COPD).

The four different conditions were: 1) through a cuff inflated cannula (condition A); 2) through the mouth with a deflated cannula (condition B); 3) through the mouth with a phonetic uncuffed cannula (condition C); and 4) through the mouth after stoma closure (condition D). Five trials in each condition were performed using a standardised method.

The measurement of both MIP and MEP differed significantly depending on the condition of measurement. MIP taken in condition A was significantly higher when compared with conditions $B, C$ and D. MEP in condition A was significantly higher when compared with condition B and D. In condition A the highest frequency of the best measurement of MIP and MEP was observed at the fourth and fifth effort, respectively. The same results were obtained after the selection of only COPD patients.

In conclusion, respiratory muscle assessment differs significantly depending on measurement condition. Measurement through inflated cannula tracheotomy yields higher values of both maximal inspiratory and maximal expiratory pressure.

KEYWORDS: Maximal expiratory pressure, maximal inspiratory pressure, tracheotomy, weaning

$\mathbf{M}$ easurement of maximal inspiratory pressure (MIP) and maximal expiratory pressure (MEP) is the most widely used test to assess muscle pressures, as it has no adverse effects, is noninvasive and relatively easy to perform [1]. Although this test has possible limitations mainly due to no cooperation or noncompliance, several studies have demonstrated that MIP and MEP may be very useful in the diagnosis and follow-up of pulmonary and cardiac disease $[2,3]$. A reduction in MIP has been shown to be associated with a progressive clinical worsening in patients affected with myasthenia gravis [4] or Guillain-Barré syndrome [5]. In addition, it has been suggested that an imbalance between the pressure generated by the inspiratory muscles and the MIP may lead to the development of hypercapnia in patients with chronic obstructive pulmonary diseases (COPD) [6]. Indeed, both MIP and MEP have been shown to be useful in detecting the presence of iatrogenic myopathy, such as steroid-induced myopathy [7] or in predicting the post-operative pulmonary complications following coronary artery by-pass graft surgery [8]. All of the aforementioned studies have been conducted in patients breathing through their natural airways. An imbalance between respiratory mechanical load and respiratory muscle capability is also a frequent cause of difficulty when weaning the patients from mechanical ventilation. Therefore, a tracheotomy is usually performed 14-21 days after intubation [9]. In these patients, the MEP has been shown to provide a reliable estimate of cough efficacy and efficient airways clearance [10]. Likewise, MIP is a very sensitive predictive index of successful weaning even though its specificity is rather low [11].

As far as the current authors are aware, no studies have verified the hypothesis that different sites of pressure recordings (i.e. at the mouth or directly at the stoma), and breathing conditions (i.e. inflated or deflated cannula), may affect measurements of both MIP and MEP in tracheotomised patients.

Therefore, the primary aim of the present study was to find differences, if any, in the measurement
AFFILIATIONS

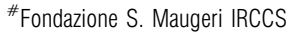

Pavia, and

${ }^{*}$ S. Maugeri Foundation Centre of Gussago-Lung Function Unit,

Gussago (BS),

๑Villa Pineta Fondazione Onlus,

Gaiato (Mo), and

+Azienda Ospedaliera di Padova,

Padova, Italy.

CORRESPONDENCE

M. Vitacca

S. Maugeri Foundation IRCCS

Pulmonary Unit

Weaning Centre

Via Pinidolo 23-I-25064 Gussago

(BS)

Italy

Fax: 390302521718

E-mail:mvitacca@fsm.it

Received:

January 102005

Accepted after revision:

October 052005 
of respiratory pressures in tracheotomised patients who are difficult to wean, depending on the site and breathing conditions of measurement. The secondary aim of the study was to assess whether an underlying diagnosis would affect the results of the measurements.

\section{METHODS}

The present study was conducted in accordance with the Declaration of Helsinki, and all hospital Ethics Committees approved the protocol. All patients gave informed consent after specific information on tests, its clinical utility and on modality to perform the measurements.

\section{Patients}

The current study reports the results of a prospective, observational study carried out on patients admitted to one of four weaning centres in Gussago, Pavia, Padova and Gaiato (Italy) from September 1, 2003 to March 31, 2004. The weaning centres of Gussago, Gaiato and Pavia are units of Rehabilitation Institutions, which are referral rehabilitation and chronic care centres for a large geographical area in Northern Italy. Among others, long-term mechanically ventilated patients are also admitted to these institutions to undergo a programme of progressive discontinuation of mechanical ventilation and to be discharged to a home programme of longterm ventilatory assistance, if liberation from the ventilator fails. The weaning centre of Padova is part of a General Regional Teaching Hospital.

In total, 38 patients had been transferred to the weaning centre from intensive care units (ICUs) of other hospitals because they had been classified by the caring physicians as difficult-towean patients. This was due to a consequence of repeated failures of weaning attempts and for that reason had undergone tracheotomy. Diagnosis was COPD in 28 patients, cardiosurgical sequel in five patients, neuromuscular in three and chest wall disease in two patients. Diagnosis of COPD was made according to the American Thoracic Society (ATS) criteria [12] based on the clinical history, physical examination, chest radiography and previous pulmonary function tests. The time elapsed from intubation to tracheotomy ranged from 3-12 days with time from intubation to weaning centre admission ranging from 18-35 days.

At the time of the evaluation all patients were in stable conditions as assessed by: 1) arterial $\mathrm{pH}>7.35$ during spontaneous breathing with an inspiratory oxygen fraction $\left(\mathrm{FI}, \mathrm{O}_{2}\right)$ able to maintain arterial oxygen saturation $\left(\mathrm{Sa}_{\mathrm{a}} \mathrm{O}_{2}\right)>90 \%$ (range 28-35\%); 2) absence of severe dyspnoea or signs of respiratory distress, such as abdominal paradox or use of accessory muscles; and 3) haemodynamic stability (systolic arterial blood pressure $>100$ and $<150 \mathrm{mmHg}$ with, no need of i.v. vasopressor drugs).

Mechanical ventilation had been withdrawn before the study. All patients were still tracheotomised, but they had been breathing spontaneously for $9 \pm 5$ days. In their stable state, before the episode of acute respiratory failure that led to ICU hospitalisation occured, $60 \%$ of patients were on long-term oxygen therapy, whereas $26 \%$ were on domiciliary long-term mechanical ventilation $(90 \%$ with mask positive pressure ventilation, the remaining through tracheotomy).
The exclusion criteria of the present study were: 1) alteration of consciousness; 2) poor comprehension of or poor collaboration with test manoeuvres; 3) contraindications to cannula removal due to anatomical deformities demonstrated by endoscope; or 4) refusal to give informed consent to the study.

\section{Measurements}

The following data were recorded at the time of the study: anthropometrics, nutritional status (weight), and length of stay in ICU.

Dynamic lung volumes were calculated by means of a mass flow sensor (VMax 22C; Sensor Medics, Yorba Linda, CA, USA) before discharge, where possible (and when the patients were already decannulated); otherwise historical data were considered, if available. The predicted values were those by QUANJER [13].

Arterial blood gases were assessed by means of an analyser (RapidLab 865; Bayer Corporation, East Walpole, MA, USA) on blood samples drawn on the day of the study, from the radial artery, while patients breathed room air.

As recently suggested [1], MIP and MEP tests were performed by an experienced operator, who strongly urged the subjects to make maximum inspiratory (Mueller manoeuvre) and expiratory (Valsalva manoeuvre) efforts at or near residual volume and total lung capacity, respectively. The measurements were conducted with a fixed and well known protocol from all the employed physiotherapists in the weaning centre. Prior to the manoeuvre the patients were educated on the modality of execution of the test, while the therapist mimed the same test to make it more comprehensible.

As these are unfamiliar manoeuvres, careful instructions and standardised encouraged motivation were given. Furthermore, MIP was obtained by coaching the patients to breathe tidally and, after a few normal breaths, to exhale as far as possible before inspiring against the occluded airway as strong and as long as possible. Conversely, to measure MEP, after a few normal breaths, patients were coached to take a deep breath before expiring as strongly as possible.

Participants were instructed to exert maximal inspiratory and expiratory effort during each measurement to maintain pressure level for at least $2 \mathrm{~s}$. This was determined by the use of a stop watch. The respiratory therapist asked patients to look at the needle of the pressure gauge as visual feed-back. No pressure time course for time tracing was adopted during the study measurements. Five consecutive efforts were performed and recorded allowing a 1-min pause between each effort.

Patients were in a sitting position, connected to a portable differential pressure transducer $\left( \pm 300 \mathrm{cmH}_{2} \mathrm{O}\right.$; Honeywell manometers, Freeport, IL, USA), breathing through a oneway valve with a tube-type piece with a small hole preventing closure of the glottis. This was then connected to the cannula, or to a flanged mouthpiece. A nose-clip was applied when using a mouthpiece. When using the mouthpiece, subjects were coached to prevent air leaks around it and to support the cheeks during the expiratory efforts, thus, helping them to pinch their lips around the mouthpiece.

Oxygen was supplied as required to achieve a pulse oxymeter $\mathrm{Sa}_{1} \mathrm{O}_{2}$ reading of $>90 \%$. The pressure measuring system was calibrated every 4 days with a water manometer. 
The best value achieved of the five peak pressure efforts was used for statistical analysis. All the measurements were performed by respiratory therapists usually in charge in the weaning centre.

\section{Protocol}

The study was conducted on two different days. The four conditions under study shown in figure 1 are as follows. 1) Through a cuff inflated cannula (condition A), MIP and MEP were measured directly through the stoma (fig. 1a) with the inner balloon cuffed and without any inner tube. 2) Through the mouth with the same cannula as in condition A with the inner balloon deflated (condition B) and without any inner tube. MIP and MEP were measured through the mouth (fig. 1b) simultaneously occluding the external hole of the cannula by a therapist's finger. The respiratory therapist was confident that the seal was complete if patients were able to speak. Particular attention was also paid to avoid any possible leak from the cannula or around the cannula itself. 3) Through the mouth with a phonetic uncuffed cannula (Biesalsky ${ }^{\circledR}$ Rüsch, Kemen, Germany; condition C). MIP and MEP were measured directly through the mouth (fig. 1c) with a phonetic uncuffed cannula and occluding the external hole of the cannula with a specific phonetic cap. This cannula presented an inner tube inside. 4) Through the mouth after closure of the stoma (condition D). MIP and MEP were measured directly through the mouth (fig. 1d) $1 \mathrm{~h}$ from decannulation. The tracheotomy wound was covered with an adhesive support. A soft manual pressure was maintained by another operator throughout the measurement, to avoid any possible leak. A respiratory therapist was confident that the seal was complete if patients were able to speak. Only after this test were the measurements performed.

Inflated, deflated and uncuffed cannulas were chosen so as to maintain the same inner diameter. No fenestrated cannulas were used during the study.
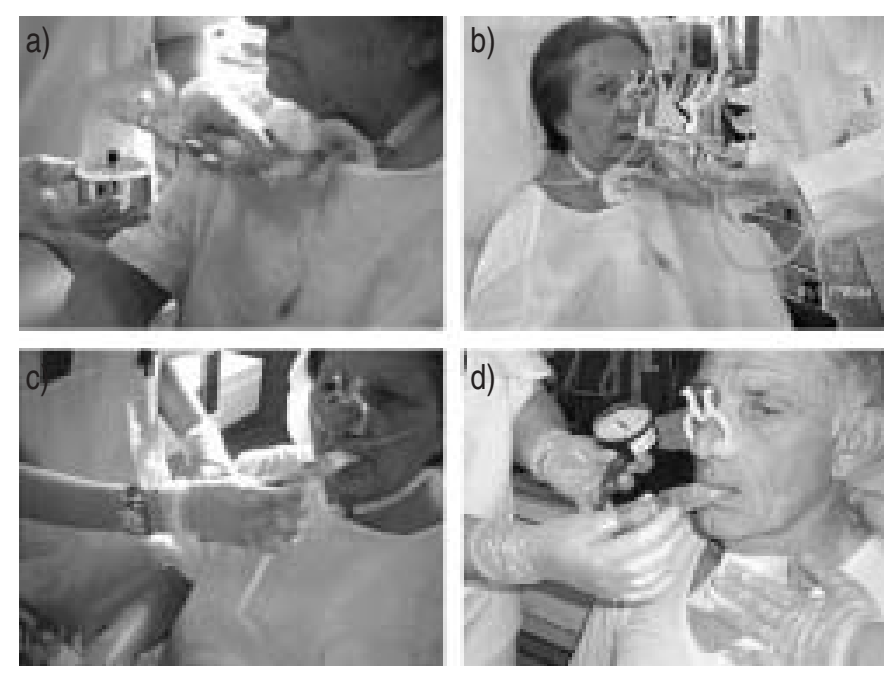

FIGURE 1. A representative patient under the four studied conditions. a) Through a cuff inflated cannula (condition A). b) Through the mouth with the same cannula (as in the first condition) with the inner balloon deflated (condition B). c) Through the mouth with a phonetic uncuffed cannula (Biesalky ${ }^{\circledR}$ Rüsch; condition C). d) Through the mouth after closure of the stoma (condition D).
Condition D was not performed if MIP and MEP measured in other conditions were $<20$ and $>30 \mathrm{cmH}_{2} \mathrm{O}$, respectively. This was because patients were considered too weak to be safely decannulated. A 1-h pause was always allowed between the two conditions studied in each day. Patients showing clinical signs of respiratory failure relapse, desaturation $<89 \%$ in spite of increasing oxygen supply $\left(\mathrm{FI}_{1} \mathrm{O}_{2}>40 \%\right)$ or refusing to complete the study were considered drop-outs.

\section{Statistical analysis}

All the results are shown as mean $\pm \mathrm{SD}$ or frequencies, when appropriate.

ANOVA for repeated measures with one factor within subjects (four levels, i.e. four different conditions of measurement) assuming an equal pair-wise correlation between groups was carried out. There was no evidence that the assumptions were not met. The highest value out of five efforts in measurement of both MIP and MEP has been considered for analysis. Bonferroni's adjustment for multiple pair-wise comparisons has been applied where appropriate. A p-value $<0.05$ was considered to be statistically significant.

\section{RESULTS}

From the 50 enrolled patients, five subjects were excluded from the study due to lack of cooperation and seven due to the presence of anatomical tracheal deformities. The remaining 38 consecutive tracheostomised patients were then considered for analysis. Table 1 shows the anthropometric data of the 38 consecutive tracheotomised patients. Of these, 23 were from the centre in Gussago, six from Padova, five from Gaiato and four from the Pavia centre.

\section{Comparison of the four different sites and conditions of measurement}

The results of the measurement of MIP and MEP, in the four conditions under study, are shown in figure 2.

ANOVA showed a significant difference $(p<0.001)$ of the measure of MIP depending on the condition of measurement. The measure of MIP taken at the cannula with the cuff inflated (condition A) was significantly higher when compared with the measurement at the mouth with the cuffed cannula and the inner balloon deflated (condition B: pairwise comparison with Bonferroni's adjustment versus $\mathrm{A} ; \mathrm{p}<0.001$ ), to the measure at the mouth with a phonetic uncuffed cannula in site (condition $C$ versus $A$; adjusted $p<0.001$ ) and to the measure taken at the mouth after closure of the stoma (condition D versus $\mathrm{A}$; adjusted $\mathrm{p}=0.012$; fig. 2a).

Similarly, ANOVA showed a significant difference $(\mathrm{p}<0.001)$ of the measure of MEP depending on the condition of measurement. MEP measured at the cannula with the cuff inflated (condition A) was significantly higher when compared with the measurement at the mouth with the cuffed cannula and the inner balloon deflated (condition B: pairwise comparison with Bonferroni's adjustment versus condition $\mathrm{A} ; \mathrm{p}=0.005$ ) and to the measurement taken at the mouth after closure of the stoma (condition D versus A; adjusted $\mathrm{p}=0.049$ ). No statistical difference was reached in the pairwise comparison between condition $\mathrm{A}$ and condition $\mathrm{C}$ (measurements taken at the 


\begin{tabular}{|c|c|}
\hline \multicolumn{2}{|c|}{ Characteristics of the study population } \\
\hline Subjects $n$ & 38 \\
\hline Males & 30 \\
\hline Age yrs & $70 \pm 12$ \\
\hline \multicolumn{2}{|l|}{ Diagnosis ${ }^{\#}$} \\
\hline COPD & 28 \\
\hline Post-cardiosurgical event & 5 \\
\hline Neuromuscular & 2 \\
\hline Chest wall diseases & 3 \\
\hline \multicolumn{2}{|l|}{ Causes of ICU admission } \\
\hline Pneumonia & 15 \\
\hline COPD exacerbations & 10 \\
\hline Cardiogenic pulmonary oedema & 3 \\
\hline Sepsis & 3 \\
\hline Acute respiratory failure & 7 \\
\hline Time from tracheotomy days ${ }^{\#}$ & $16 \pm 3$ \\
\hline Length of ICU stay days ${ }^{\#}$ & $24 \pm 4$ \\
\hline Duration of MV days & $29 \pm 4$ \\
\hline Cannula diameter at admission $\mathrm{cm}^{\circ}$ & $7.5 \pm 0.75$ \\
\hline \multicolumn{2}{|l|}{ Type of cuffed cannula at study entry } \\
\hline Mallinkrodt & 9 \\
\hline Shiley & 6 \\
\hline Portex blue line & 22 \\
\hline Bivona & 1 \\
\hline FEV $_{1} \%$ pred $^{+}$ & $28 \pm 6$ \\
\hline FVC \% pred ${ }^{+}$ & $34 \pm 7$ \\
\hline $\mathrm{pH}^{*}$ & $7.38 \pm 0.02$ \\
\hline $\mathrm{Pa}, \mathrm{O}_{2} / \mathrm{FI}_{1} \mathrm{O}_{2} \mathrm{C}$ & $267 \pm 45$ \\
\hline $\mathrm{Pa}, \mathrm{CO}_{2} \mathrm{mmHg}^{\circ}$ & $50 \pm 5$ \\
\hline
\end{tabular}

Data are presented as mean \pm SD or $\mathrm{n}$. COPD: chronic obstructive pulmonary disease; ICU: intensive care unit; MV: mechanical ventilation; FEV1: forced expiratory volume in one second; FVC: forced vital capacity; $\mathrm{Pa}_{\mathrm{a}} \mathrm{O}_{2}$ : arterial oxygen tension; $\mathrm{Fl}, \mathrm{O}_{2}$ : inspiratory oxygen fraction; $\mathrm{Pa}_{1} \mathrm{CO}_{2}$ : carbon dioxide arterial

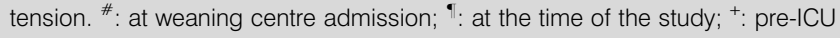
admission, or before rehabilitative hospital discharge. $\mathrm{mmHg}=0.133 \mathrm{kPa}$.

mouth with a phonetic uncuffed cannula in site; adjusted $\mathrm{p}=0.43$; fig. $2 b)$.

The frequency of the best within subject's measurement of MIP and MEP among the five repeated efforts is shown in figure 3. The highest frequency of the best measurement of MIP was observed in the fifth effort in all of the conditions, except in condition A (i.e. at the cannula with the cuff inflated) where the best value was more frequently observed at the fourth effort. The best measure has seldom been observed in the first two efforts, in all of the conditions.

Likewise, during the MEP measurments, the highest value was more frequently observed in the fifth effort, in all of the conditions, compared particularly with the first and second effort.

\section{Influence of the diagnosis}

To rule out any confounding effect on the results, derived from the heterogeneity of diagnosis of the patients in the study, all patients with non-COPD diagnosis were excluded and the main analysis was only repeated in the COPD population (28
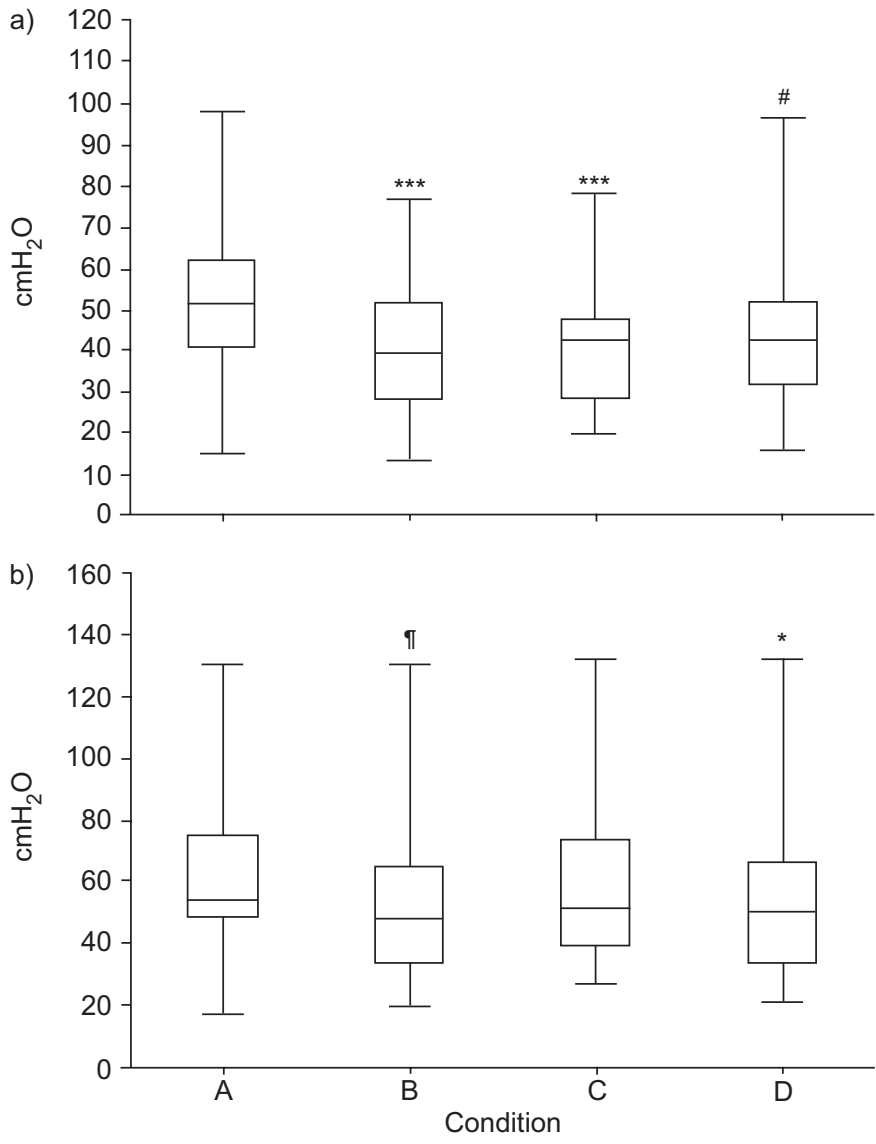

FIGURE 2. Effect of different modality/site of measurement on a) maximal inspiratory pressure and b) maximal expiratory pressure. The best values were chosen among five repeated manoeuvres in each modality. A-D correspond to the conditions described in figure 1. Boxes represent the inter-quartile range (25-75\%) with the line representing the median. The bar lines represent minimum-maximum values. ${ }^{* *}: p<0.001 ;{ }^{*}: p=0.012$, Bonferroni's adjustment for pairwise comparison; ${ }^{\uparrow}: p=0.005 ; *: p=0.05$.

out of 38 patients). The same results as in the whole study sample were observed. The measurement of MIP was significantly different (ANOVA $p<0.001$ ) among the four conditions. The value of MIP measurement was higher at the cannula with cuff inflated (condition A) when compared with the other conditions (pairwise comparisons with Bonferroni's adjustment $\mathrm{p}<0.001, \mathrm{p}=0.001$ and $\mathrm{p}<0.001$ versus conditions $\mathrm{B}$, $\mathrm{C}$ and $\mathrm{D}$, respectively). Measurement of MEP was significantly different (ANOVA $\mathrm{p}<0.001$ ) among the four conditions. MEP was higher when measured in condition $A$ and compared with condition B (adjusted $\mathrm{p}=0.005$ ) and $\mathrm{D}$ (adjusted $\mathrm{p}=0.03$ ), but not when compared with condition $C$ (adjusted $\mathrm{p}=0.147)$.

\section{Outcome of decannulation}

All patients were safely decannulated 30 days after the study. Due to a relapse of their disease, two patients needed a further period of mechanical ventilation which was successfully administered noninvasively. Six out of the 38 patients investigated $(16 \%)$ were discharged with nocturnal home noninvasive mechanical ventilation. 

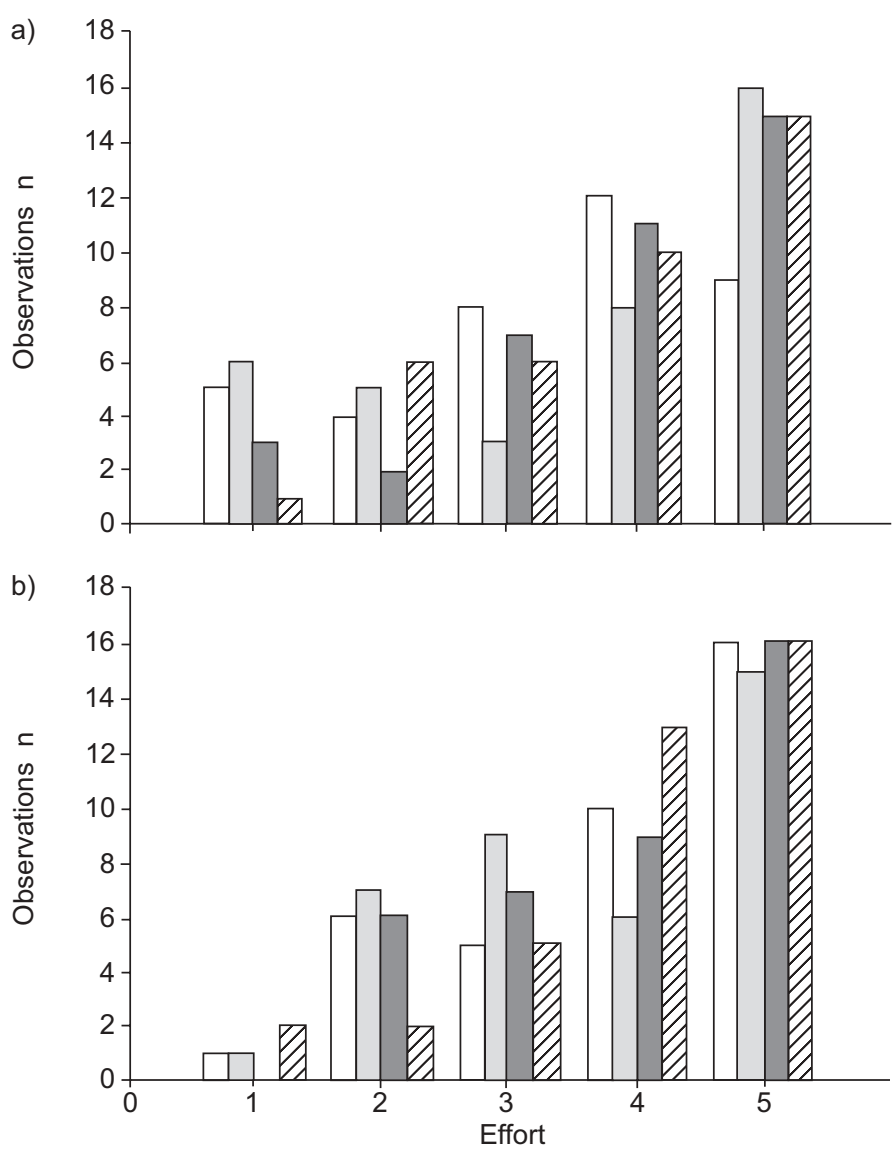

FIGURE 3. Frequency of the best within subject's measure of a) maximal inspiratory pressure and $b$ ) maximal expiratory pressure among the five repeated efforts in each modality/site of measurement. $\square$ : A; $\square$ : B; $\square$ : C; $\mathbb{Z}$ : D. A-D correspond to the conditions described in the Protocol section.

\section{DISCUSSION}

The present study demonstrates that the assessment of respiratory muscles' pressures in critically ill tracheotomised patients differs significantly, depending on the measurement condition. In particular, the measurement through tracheotomy, with inflated cuff, yields higher values of both MIP and MEP, when compared with the other conditions. The highest value of the five efforts in each condition was more frequently observed in the last attempts of measurement, thus, indirectly demonstrating a learning effect of the manoeuvre. These results, obtained in a miscellaneous sample of difficult-towean tracheotomised patients, were not significantly different from when only the patients with COPD diagnosis were selected.

Several studies have demonstrated that the measurement of respiratory muscle force by means of MIP and MEP may be very useful in the diagnosis and follow-up of pulmonary and cardiac diseases [2, 3], as well as in patients affected with myasthenia gravis [4] or Guillain-Barré syndrome [5].

Likewise, the finding of abnormally low maximal respiratory pressures is able to predict the onset of post-operative pulmonary complications in patients undergoing coronary artery by-pass graft surgery [8]. However, in COPD and asthma patients who have undergone prolonged steroid treatment, it is highly suggestive that there is a presence of iatrogenic myopathy [7]. The pressure generated by the respiratory muscles to MIP ratio (inspiratory pressure/maximal pressure) has been shown to be significantly impaired in patients with COPD [6]. Both the reduced capacity of the respiratory muscle (i.e. force) and an increased respiratory load (i.e. elastic and resistive) variably contribute to the development of respiratory muscle fatigue and to the impossibility of successfully sustaining spontaneous breathing in the critically ill patient. In this particular setting, it is of utmost importance that a reliable assessment of generated respiratory pressures constitutes, as far as possible, a real estimate of respiratory muscle force. The reliability of these measurements, which is highly dependent upon volitional effort, may be significantly reduced, particularly where lack of collaboration and compliance exist. This may represent a frequent case in the ICU setting, where patients with altered consciousness are admitted and sedation is often used. This also happens in the respiratory intermediate ICU. In fact, in the present study a quarter of patients didn't fulfil the inclusion criteria for enrolment in the study.

Despite the aforementioned limitations, respiratory muscle force assessment by measuring maximal respiratory pressures have been proposed, among others, as useful indices for decision making for extubation [14, 15], discontinuation from mechanical ventilation or removal of tracheal cannula [16] and assessing cough efficiency $[10,17]$ to start a specific rehabilitative programme $[18,19]$ as well as to assess its effectiveness [18].

Although a standardised approach to measurement of respiratory muscles strength and endurance has been proposed by the ATS and European Respiratory Society (ERS) [1], demographic and anthropometrical factors may variably contribute to the range of the normal values in healthy adults [20-26]. Methodological factors may account for measurement variability among different subjects with possible confusing factors which contribute to the difficulty of both measurement and interpretation of respiratory muscles function $[1,17,18,22,26-$ 29]. Finally, and more importantly, no particular technical issues and detailed instructions have been recommended by the ATS and ERS document regarding when and where respiratory pressures should be measured in patients not breathing through their natural airways.

In a questionnaire survey conducted among respiratory therapists from nine hospitals, SOO HOO and PARK [15] showed that, despite its clinical usefulness in the weaning process, a wide range of variation resulted from measurement of MIP by respiratory therapists in an ICU setting. Major issues were concerned with methods of obtaining respiratory pressures, lack of occlusion of the airway for the recommended amount of time, reproducibility, interpretation of the maximal effort and test-to-test variation. In that survey, $87 \%$ of respiratory therapists used a manometer, 38\% using the ventilator's software and $25 \%$ using both of them [15]. Moreover, the duration of airway occlusion time ranged from $2-4 \mathrm{~s}$ in $56 \%$ of the cases. Finally, $68 \%$ of respiratory therapists performed at least three measurements, with $75 \%$ of them only recording the highest value [15]. The ATS/ERS [1] document suggested MIP 
measurement in intubated patients with poor cooperation should be performed using the so-called Marini method [30], whose reproducibility has been subsequently shown to be poor. MARINI et al. [30] used a unidirectional valve to allow the output, but not the input of air. They also measured MIP starting from residual volume (RV) and performed 15-20 consecutive tests after 15-20 s of airway occlusion. In patients under mechanical ventilation, SPRAUGE and HOPKINS [31] measured MIP using five to 10 consecutive repetitions, starting from RV and recording the average value among three. BRUTON [32] performed the measurement according to the operator's decision and then recorded only the highest value. CARUSO et al. [33] did not describe the method employed, while EL-KHATIB et al. [34] performed three tests and recorded the average. No uniformity exists in the measurement of maximal respiratory pressures in the intensive care setting and in particular in difficult-to-wean and consequently tracheotomised patients. It is obvious that any parameter can only be useful if it accurately reflects the patient's condition. Therefore, a call for further standardisation of measurement of respiratory pressures in these patients is surely needed.

The present study is the first, to the authors' knowledge, that assesses maximal respiratory pressure measurement in different patient's conditions, at the same time evaluating the effect of different tracheotomy cannulas and breathing conditions when compared with after-stoma closure.

The highest values of both MIP and MEP were obtained with the direct measurement at the cannula with the cuff inflated (condition A). This evidence is not surprising and is possibly explained by the fact that direct measurement of pressures at the trachea most likely resembles maximal oesophageal $[35,36]$ or cough gastric pressures measured by means of oesophageal and gastric balloons. In particular, clinical situations in which standard measurement of respiratory pressures is difficult or scarcely reliable, such as in patients with facial muscle weakness or bulbar dysfunction [37, 38], maximal sniff manoeuvre and cough pressures, are considered accurate and reproducible estimates of respectively inspiratory and expiratory muscle strength. Moreover, in the measurement at the trachea with the cuff inflated, air leaks can be reasonably ruled out. It is no surprise, then, that no statistically significant differences were otherwise observed among the other conditions studied. Despite a rigorous standardised procedure in the measurement of respiratory pressures, air leaks around the cannula, insufficient sealing by the therapist's finger occluding the cannula (when measuring at the trachea) or collapse of the upper airways (when measuring at the mouth) cannot be theoretically excluded. However, it seems that air leaks would be a major concern mainly in the measurement of MIP rather than MEP. Multiple comparisons among different conditions demonstrate lower levels of significance when measuring expiratory pressures than when measuring inspiratory pressures. The lack of statistical significance between the measure at the cannula with the cuff inflated and the measure at the mouth with the phonetic cannula would further confirm this observation.

As previously outlined, the number of repetitions of the measurement is extremely variable in the literature, ranging from three to 20 in the different studies. In the present study, the highest value of the measurement in each condition, both of MIP and MEP, was rarely observed in the first efforts. In contrast, the best performance was most frequently observed in the last two efforts, thus, suggesting a learning effect of the manoeuvre. A further improvement of the performance with increasing the number of efforts cannot be excluded. In fact, a major limitation of the present study is that the decision was made to set the limit to five efforts in each condition of study for both MIP and MEP measurement. On one hand, the current authors decided more efforts than in usual practice were needed (when respiratory therapists usually consider the best of three consecutive efforts), but on the other hand did not want to risk producing fatigue. In fact, a previous pilot study conducted on a limited sample of patients, such as those enrolled for the present protocol, had shown a lack of cooperation with more than five efforts. Other limitations of the study were that the respiratory therapists were not blinded to the study and those measurements were not recorded on a computer or a paper tracing.

In conclusion, the present study shows that respiratory muscles assessment differs significantly only when measurement is performed through the tracheotomy with the cuffed cannula, which yields the highest values of both maximal inspiratory pressure and maximal expiratory pressure measurements. Values of both maximal inspiratory pressure and maximal expiratory pressure obtained in all the other studied conditions of measurement (i.e. through the mouth with the cuff deflated and the cannula occluded, through the mouth with a phonetic cannula and, conventionally, through the mouth after stoma closure) were not statistically different. Finally, in this particular setting of patients, at least five measurements could be taken as a reasonable compromise between the possibility to achieve a better performance due to a learning effect and the risk of onset fatigue and lack of cooperation.

\section{REFERENCES}

1 ATS/ERS statement on respiratory muscle testing. Am J Respir Crit Care Med 2002; 166: 518-624.

2 Meyer FJ, Lossnitzer D, Kristen AV, et al. Respiratory muscle dysfunction in idiophatic pulmonary arterial hypertension. Eur Resp J 2005; 25: 125-130.

3 Lavietes $\mathrm{MH}$, Gerual CM, Fless KG, et al. Inspiratory muscle weakness in diastolic dysfunction. Chest 2004; 126: 838-844.

4 Munoz Fernandez C, Diez Tejedor E, Garcia F, et al. Evaluation of maximal respiratory pressures in myasthenia gravis. Prognostic value. Eur Neurol 2004; 52: 136-140.

5 Cheng BC, Chang WN, Chang CS, et al. Predictive factors and long-term outcome of respiratory failure after Guillain-Barre syndrome. Am J Med Sci 2004; 327: 336-340.

6 Begin P, Grassino A. Inspiratory muscle dysfunction and chronic hypercapnia in COPD. Am Rev Respir Dis 1991; 143: 905-912.

7 Decramer M, Stas KJ. Corticosteroids induced myopathy involving respiratory muscles in patients with chronic obstructive pulmonary disease and asthma. A Rev Respir Dis 1992; 146: 800-802. 
8 Hulzebos EH, Van Meeteren NL, De Bie RA, et al. Prediction of postoperative pulmonary complications on the basis of preoperative risk factors in patients who had undergone coronary artery bypass graft surgery. Phys Ther 2003; 83: 8-16.

9 Fischler L, Erhart S, Leger GR, Frutiger A. Prevalence of tracheostomy in ICU patients. A Nation-wide survey in Switzerland. Intensive Care Med 2000; 26: 1428-1433.

10 Chatwin M, Ross E, Hart N, Nickol AH, Polkey MI, Simonds AK. Cough augmentation with mechanical insufflation/exsufflation in patients with neuromuscular weakness. Eur Respir J 2003; 21: 502-508.

11 Meade M, Guyatt G, Cook D, et al. Predicting success in weaning from mechanical ventilation. Chest 2001; 120: Suppl. 6, 400S-424S.

12 American Thoracic Society Statement. Standards for the diagnosis and care of patients with chronic obstructive pulmonary disease. Am J Respir Crit Care Med 1995; 152: S77-S120.

13 Quanjer PH. Working Party on "Standardization of lung function tests". Bull Eur Physiopathol Respir 1983; 19: Suppl. 5, 7-10.

14 MacIntyre NR, Cook DJ, Ely EW Jr, et al., Chest 2001; 120: Suppl. 6, 375S-395S.

15 Soo Hoo GW, Park L. Variations in the measurement of weaning parameters. Chest 2002; 121: 1947-1955.

16 Ceriana P, Carlucci A, Navalesi P, et al. Weaning from tracheotomy in long-term mechanically ventilated patients: feasibility of a decisional flow weaning centre chart and clinical outcome. Intensive Care Med 2003; 29: 845-848.

17 Badr C, Elkins MR, Ellis ER. The effect of body position on maximal expiratory pressures and flow. Aust J Physiother 2002; 48: 95-102.

18 Nava S. Rehabilitation of patients admitted to a respiratory intensive care unit. Arch Phys Med Rehabil 1998; 79: 849-854.

19 Stiller K. Physiotherapy in intensive care. Towards and evidence-based practice. Chest 2000; 118: 1801-1813.

20 Black LF, Hyatt RE. Maximal respiratory pressures: normal values and relationship to age and sex. Am Rev Respir Dis 1969; 99: 696-702.

21 Harik-Khan RI, Wise RA, Fozard JL. Determinants of maximal inspiratory pressure. The Baltimore Longitudinal Study of Aging. Am J Respir Crit Care Med 1998; 158: 1459-1464.

22 Bruschi C, Cerveri I, Zoia MC, et al. Reference values of maximal respiratory mouth pressures: a population-based study. Am Rev Respir Dis 1992; 146: 790-793.

23 Karvonen J, Saarelainen S, Nieminem MM. Measurement of respiratory muscle forces based on maximal inspiratory and expiratory pressures. Respiration 1994; 61: 28-31.

24 Hautmann H, Hefele S, Schotten K, Huber RM. Maximal inspiratory mouth pressures (PIMAX) in healthy subjects - what is the lower limit of normal? Respir Med 2000; 94: 689-693.

25 McConnell AK, Copestake AJ. Maximum static respiratory pressures in healthy elderly men and women: issues of reproducibility and interpretation. Respiration 1999; 66: 251-258.

26 Rubinstein I, Slutsky AS, Rebuck AS, et al. Assessment of maximal expiratory pressure in healthy adults. J Appl Physiol 1988; 64: 2215-2219.

27 Fiz JA, Carreras A, Aguilar J, Gallego M, Morera J. Effect of order on the performance of maximal inspiratory and expiratory pressures. Respiration 1992; 59: 288-290.

28 Wohlgemuth M, van der Kooi EL, Hendriks JC, Padberg GW, Folgering HT. Face mask spirometry and respiratory pressures in normal subjects. Eur Respir J 2003; 22: 1001-1006.

29 Windisch W, Hennings E, Sorichter S, Hamm H, Criee CP. Peak or plateau maximal inspiratory mouth pressure: which is best? Eur Respir J 2004; 23: 708-713.

30 Marini JJ, Smith TC, Lamb V. Estimation of pressure muscle strength in mechanically ventilated patients: the measurement of maximal inspiratory pressure. J Crit Care 1986; 1: 32-38.

31 Sprague SS, Hopkins PD. Use of pressure strength training to wean six patients who were ventilator-dependent. Phys Ther 2003; 83: 171-181.

32 Bruton A. A pilot study to investigate any relationship between sustained maximal inspiratory pressure and extubation outcome. Heart Lung 2002; 31: 141-149.

33 Caruso P, Friedrich C, Denari S. The unidirectional valve is the best method to determine maximal inspiratory pressure during weaning. Chest 1999; 115: 1096-1101.

34 El-Khatib MF, Baumeister B, Smith PG, Chatburn RL, Blumer JL. Inspiratory pressure/maximal inspiratory pressure: does it predict successful extubation in critically ill infants and children? Intensive Care Med 1996; 22: 264-268.

35 Mills GHJ, Ponte J, Hamnegard $\mathrm{CH}$, et al. Tracheal tube pressure change during magnetic stimulation of the phrenic nerves as an indicator of diaphragm strength on the intensive care unit. Br J Anaesth 2001; 87: 876-884.

36 Watson AC, Hughes PD, Louise Harris M, et al. Measurement of twitch transdiaphragmatic, esophageal, and endotracheal tube pressure with bilateral anterolateral magnetic phrenic nerve stimulation in patients in the intensive care unit. Crit Care Med 2001; 29: 1325-1331.

37 Laroche CM, Mier AK, Moxham J, Green M. The value of sniff esophageal pressures in the assessment of global pressure muscle strength. Am Rev Respir Dis 1988; 138: 598-603.

38 Man WDC, Kyroussis D, Fleming TA, et al. Cough gastric pressure and maximum expiratory mouth pressure in humans. Am J Respir Crit Care Med 2003; 168: 714-717. 